\title{
ALL 3-MANIFOLDS IMBED IN 5-SPACE
}

\author{
BY C. T. C. WALL
}

Communicated by E. Spanier, January 6, 1965

It has been shown by Hirsch [4, Theorem 4.6] that any nonclosed smooth $n$-manifold can be imbedded in $R^{2 n-1}(n \geqq 1)$. Also, Haefliger and Hirsch [2, Theorem 1.1] proved that if $n>4$, a smooth closed $n$-manifold can be imbedded in $R^{2 n-1}$ if and only if its normal StiefelWhitney class $\bar{W}^{n-1}$ vanishes (and, according to Massey and Peterson [10, Lemma 9], $\bar{W}^{n-1}$ can only be nonzero if the manifold is nonorientable and $n$ a power of 2). As to the cases $n \leqq 4$, the imbedding is clearly impossible for $n \leqq 1$; it can be done for $n=2$ (here, $\bar{W}^{1}$ vanishes precisely in the orientable case) classically. In this note, we shall also accomplish the proof for $n=3$. Only $n=4$ remains undecided. We note that the only gap in the proof of [2] for $n=4$ is the appeal to the result of Haefliger; in the piecewise linear case this can be filled by using instead a result of Irwin [6]. (The details of this argument will appear in a paper of Hirsch.) For $n=3$, every piecewise linear manifold can be smoothed, and every paracompact manifold triangulated, so all these are included in our result. The pattern of the proof resembles that in the orientable case (settled by Hirsch in $[5])$.

All the imbedding theorems for 3-manifolds depend on the following principle, first used by Hirsch in [4].

Suppose $M$ immersed in $R^{*}$, and that $M$ has a subcomplex $S^{s}$ such that $M$ can be imbedded in any neighbourhood of $S$, and $\kappa \geqq 2 s+1$. Then $M$ can be imbedded in $\boldsymbol{R}^{\boldsymbol{k}}$.

For since imbeddings are dense in the space of maps $S^{s} \rightarrow R^{\kappa}$, and immersions are open in the space of maps $M \rightarrow R^{\star}$, we may suppose that the immersion imbeds $S$. It then imbeds some neighbourhood of $S$ (it is not necessary for this to assume $S$ compact) and the result follows.

We shall call a subcomplex $S$ as above a spine of $M$. Our proposed theorem will then follow from:

Any closed 3-manifold $M$ bounds a 4-manifold $W$ such that (a) $W$ immerses in $R^{5}$, and (b) Whas a 2-dimensional spine.

The proof of this will be split in three parts: discussion of the tangent bundle of $M$ (for completeness), cobordism theory (to obtain $W$ satisfying (a)) and surgery (to obtain also (b)).

A priori, the group of the tangent bundle of $M$ may be taken as $O_{3}$. We note two subgroups each with two elements: $O_{1}$ and the centre $Z$ 
(consisting of \pm the identity matrix). Since each contains an orientation-reversing element, each homogeneous space $O_{3} / O_{1}, O_{3} / Z$ is diffeomorphic to $\mathrm{SO}_{3}$. Thus for any 3-complex $M$, there is only one obstruction $o_{1}$ (respectively, $\left.o_{2}\right) \in H^{2}\left(M ; Z_{2}\right)$ to reducing the group of a given $O_{3}$-bundle to $O_{1}$ (respectively, $Z$ ). To calculate $o_{1}$ and $o_{2}$, we identify $B_{O_{1}}$ and $B_{Z}$ with real projective space $P_{N}(R)$; the corresponding universal bundles are $\eta+\epsilon+\epsilon, \eta+\eta+\eta$ (where $\eta$ is the nontrivial, $\epsilon$ the trivial line bundle) and have $w_{2}=0, w_{2} \neq 0$, respectively. Hence $o_{1}=w_{2}$, $o_{2}=w_{2}+w_{1}^{2}$.

Now calculate Stiefel classes of the tangent bundle to $M$ by Wu's method. We have $\sum w_{i}=\sum S q^{i} \sum v_{k}$, where $v_{i} x_{3-i}=S q^{i} x_{3-i}$ for all $x_{3-i} \in H_{3-i}(M)$. So $\sum v_{k}=1+v_{1}$, and $\sum w_{i}=1+v_{1}+v_{1}^{2}$. And $w_{2}=w_{1}^{2}$, so $o_{2}=0$ : the group of the tangent bundle reduces to $Z$. (We have not seen this result in print before.) For the normal bundle (whose group must also reduce to $O_{3}$ ), we deduce $\bar{w}_{2}=0$, so $o_{1}=0$ and the group reduces to $O_{1}$. (This result is, of course, well known.)

Next, we need the cobordism group for manifolds with $O_{1}$ as structural group of the stable normal bundle. As $B_{O_{1}}$ we can take $P_{N}(R)$. The Thom space of $\eta$ is just $P_{N+1}(R)$. If the normal bundle has dimension $n$, the group is [13] $\pi_{n+3}\left(S^{n-1} P_{N+1}(R)\right)$, where $S^{n-1}$ denotes $(n-1)$-fold suspension. According to Liulevicius [9], this group is cyclic of order 2 . Note that in the case of $S O_{1}$ we obtain $\pi_{n+3}\left(S^{n}\right)$, which is cyclic of order 24. The inclusion $S O_{1} \subset O_{1}$ induces inclusions $S^{1}=P_{1}(R) \subset P_{N+1}(R), f: S^{n} \subset S^{n-1} P_{N+1}(R)$, and a map $f_{*}: \pi_{n+3}\left(S^{n}\right)$ $\rightarrow \pi_{n+3}\left(S^{n-1} P_{N+1}(R)\right)$. We assert that $f$ is onto. Indeed, in Liulevicius' computation, the element of order 2 arises from a term $e_{01} h_{1}$ $\in \operatorname{Ext}^{1,5}\left(H^{*}\left(P_{N+1}(R)\right) ; Z_{2}\right)$ in the $E_{2}$ term of the spectral sequence of Adams [1]. But since $f$ induces a cohomology monomorphism, $e_{01}$ is the image of the generator $e_{01}^{\prime}$ of $\operatorname{Ext}^{0,1}\left(H^{*}\left(S_{1} ; \boldsymbol{Z}_{2}\right), \boldsymbol{Z}_{2}\right)$, so $f_{*}\left(e_{01}^{\prime} h_{1}\right)$ $=e_{01} h_{1}$. The result follows from naturality of the Adams spectral sequence, and the fact that all differentials vanish on $e_{01}^{\prime} h_{1}$.

We next assert that if $M^{3}$ is orientable (nonorientable) we can choose a reduction of the group of the stable normal bundle to $S O_{1}\left(O_{1}\right)$ so that $M$ bounds an $S_{1^{-}}\left(O_{1^{-}}\right)$manifold $W^{4}$. In fact, consider a 3-disc in $M$. The $S_{1^{-}}$(or $O_{1^{-}}$) structure can be defined over this disc by a framing. We change the framing by $\alpha \in \pi_{3}\left(S O_{n}\right)$. According to Kervaire [7], this changes the framed (i.e., $\mathrm{SO}_{1}$ ) cobordism class by $J \alpha$ in the orientable case; since $J: \pi_{3}\left(S_{n}\right) \rightarrow \pi_{n+3}\left(S^{n}\right)$ is onto, our assertion follows. The same is valid in the nonorientable case since the composite $f_{*} J$ is onto.

Finally, we perform surgery on $W$. First note (cf. Smale [12]) that $W$ has a presentation formed by attaching $i$-handles $(0 \leqq i \leqq 4)$ 
to $M \times I$. According to Smale [11] we may suppose the handles attached in order of increasing $i$; also $[12, \S 4]$ that there are no 0 handles. We will perform surgery to get rid of 1-handles. Now a 1handle is attached to $M$ by two discs lying in $M$. We choose an arc $\alpha$ joining the discs, and thicken it; hence the two discs lie in a single disc $\Delta$ in $M$. Attaching the 1 -handle is the same as glueing to $M$ along $\Delta$ a handlebody $\in \mathcal{H C}(4,1,1)$, i.e., a $D^{3}$-bundle over $S^{1}$. If this is nonorientable, $W$, hence also $M$, is nonorientable; we repeat the construction with $\alpha$ in the other orientation class (of arcs joining the two given discs). If it is orientable, we have $D^{3} \times S^{1}$.

We will replace this by $S^{2} \times D^{2}$; however, some care is necessary. Since $D^{3} \times S^{1}$ is orientable, even if $W$ is not, the $S O_{1}$ - or $O_{1}$-structure can be defined by a framing over it. We now perform framed surgery (Kervaire and Milnor [8, Lemma 6.2]); this ensures that the new manifold resulting from $W$ still has $\mathrm{SO}_{1}$ - or $\mathrm{O}_{1}$-structure. Also, attaching $S^{2} \times D^{2}$ to $M$ along $\Delta$ can be described by adding a 2 -handle. (This method of replacing 1 -handles by 2 -handles is due to Wallace $[15, \mathrm{p}$. $521]$.) We continue in this way by induction until no 1 -handles are left.

We assert that the resulting manifold $W^{4}$ satisfies conditions (a) and (b) required by our plan. Indeed, $W^{4}$ still has an $O_{1}$-structure on its stable normal bundle. It follows from Hirsch [3, Theorem 6.4] that $W^{4}$ immerses in $R^{5}$, i.e., (a) is satisfied. But consider the handle decomposition of $W$ dual to the one above. In this, there are only handles of dimensions 0,1 , and 2. Assertion (b), and hence our main result, now follows from

Proposition. Let $W$ be a manifold with a handle presentation with all handles of dimension $\leqq r$. Then $W$ has an r-dimensional spine.

Proof. We will use induction on the number of handles. An $r$ handle is defined by attaching $D^{r} \times D^{m-r}$ along $S^{r-1} \times D^{m-r}$. We call $D^{r} \times 0$ the core of the handle. Clearly $D^{r} \times D^{m-r}$ can be retracted, by a deformation which is an imbedding at each stage, towards $\left(S^{r-1}\right.$ $\left.\times D^{m-r}\right) \cup\left(D^{r} \times 0\right)$.

Our induction will produce a spine $S$, a map $\phi: \partial W \rightarrow S$, with mapping cylinder $M$, and a homeomorphism $\psi: W \rightarrow M$ which induces a diffeomorphism of $W-S$ on $\partial W \times[0,1)$. When we attach an $r$ handle to $W$, we add to $S$ its core, and the mapping cylinder of $\phi \mid\left(S^{r-1} \times 0\right)$. We need only redefine $\phi$ near $S^{r-1} \times D^{m-r}$, and $\psi$ correspondingly; this can easily be done in suitable local coordinates, using the remarks above.

It is, perhaps, also worth commenting that this method gives a general imbedding theorem which improves some of those referred to at the start of this paper. 
TheOREM. Suppose $M^{m}$ has boundary $\partial M$, and that the pair $(M, \partial M)$ is $r$-connected, $r \leqq m-4$. If $M$ immerses in $R^{s}$ (which happens if $s \geqq 2 m$ $-r-1)$ and $s \geqq 2 m-2 r-1$, then $M$ imbeds in $R^{s}$.

Proof. By methods of Smale and Mazur one can show that $M$ has a handle decomposition based on $\partial M$, and with no handles of dimension $\leqq r$. (See, for example, [14, Theorem 5.5].) Taking the dual decomposition, and applying the above proposition, shows that $M$ has an $(m-r-1)$-dimensional spine $S$. The result now follows from our general principle, enunciated at the start of this paper. Immersions can be constructed since, as $S$ is a deformation retract of $M$, any bundle (e.g., a normal bundle) over $M$ with fibre dimension $\geqq(m-r)$ has a cross-section.

Added in proof. Our main result has also been announced by V. A. Rohlin, Dokl. Akad. Nauk SSSR 160 (1965), 549-551.

\section{REFERENCES}

1. J. F. Adams, On the structure and applications of the Steenrod algebra, Comment Math. Helv. 32 (1958), 180-214.

2. A. Haefliger and M. W. Hirsch, On the existence and classification of differentiable embeddings, Topology 2 (1963), 129-135.

3. M. W. Hirsch, Immersions of manifolds, Trans. Amer. Math. Soc. 93 (1959), 242-276.

4. - On imbedding differentiable manifolds in Euclidean space, Ann. of Math. (2) 73 (1961), 566-571.

5. - The imbedding of bounding manifolds in Euclidean space, Ann. of Math. (2) 74 (1961), 494-497.

6. M. C. Irwin, Combinatorial embeddings of manifolds, Bull. Amer. Math. Soc. 68 (1962), 25-27.

7. M. A. Kervaire, An interpretation of $G$. Whitehead's generalisation of the Hopf invariant, Ann. of Math. (2) 69 (1959), 345-364.

8. M. A. Kervaire and J. W. Milnor, Groups of homotopy spheres. I, Ann. of Math. (2) 77 (1963), 504-537.

9. A. Liulevicius, $A$ theorem in homological algebra, and stable homotopy of projective space, Trans. Amer. Math. Soc. 109 (1963), 540-552.

10. W. S. Massey and F. P. Peterson, On the dual Stiefel-Whitney classes of a manifold, Bol. Soc. Mat. Mexicana 8 (1963), 1-13.

11. S. Smale, On gradient dynamical systems, Ann. of Math. (2) 74 (1961), 199-206.

12. - Generalised Poincaré's conjecture in dimensions greater than four, Ann. of Math. (2) 74 (1961), 391-406.

13. R. Thom, Quelques proprietes globales des varietés differentiables, Comment Math. Helv. 28 (1954), 17-86.

14. C. T. C. Wall, Differential topology. IV. Theory of handle decompositions, Mimeographed, Cambridge, 1964.

15. A. H. Wallace, Modifications and cobounding manifolds, Canad. J. Math. 12 (1960), 503-528.

Mathematical Institute, Oxford, England 